\title{
Clausenidin from Clausena excavata induces apoptosis in hepG2 cells via the mitochondrial pathway
}

\begin{abstract}
Ethnopharmacological relevance: Clausena excavata Burm.f. is used locally in folk medicine for the treatment of cancer in South East Asia.

Aim of the study: To determine the mechanism of action of pure clausenidin crystals in the induction of hepatocellular carcinoma (hepG2) cells apoptosis.

Materials and methods: Pure clausenidin was isolated from Clausena excavata Burm. $f$. and characterized using ${ }^{1} \mathrm{H}$ and ${ }^{13} \mathrm{C}$ NMR spectra. Clausenidin-induced cytotoxicity was determined by MTT assay. The morphology of hepG2 after treatment with clausenidin was determined by fluorescence and Scanning Electron Microscopy. The effect of clausenidin on the apoptotic genes and proteins were determined by real-time qPCR and protein array profiling, respectively. The involvement of the mitochondria in clausenidin-induced apoptosis was investigated using MMP, caspase 3 and 9 assays.

Results: Clausenidin induced significant $(\mathrm{p}<0.05)$ and dose-dependent apoptosis of hepG2 cells. Cell cycle assay showed that clausenidin induced a G2/M phase arrest, caused mitochondrial membrane depolarization and significantly $(\mathrm{p}<0.05)$ increased expression of caspases 3 and 9, which suggest the involvement of the mitochondria in the apoptotic signals. In addition, clausenidin caused decreased expression of the anti-apoptotic protein, $\mathrm{Bcl} 2$ and increased expression of the pro-apoptotic protein, Bax. This finding was confirmed by the downregulation of Bcl-2 gene and upregulation of the Bax gene in the treated hepG2 cells.

Conclusion: Clausenidin extracted from Clausena excavata Burm.f. is an anti-hepG2 cell compound as shown by its ability to induce apoptosis through the mitochondrial pathway of apoptosis. Clausenidin can potentially be developed into an anticancer compound.
\end{abstract}

Keyword: Clausenidin; Apoptosis; Liver cancer; MMP; Bcl 2; Bax 